\title{
Lifting valid inequalities for the precedence constrained knapsack problem
}

Citation for published version (APA):

van de Leensel, R. L. J. M., van Hoesel, C. P. M., \& van de Klundert, J. (1997). Lifting valid inequalities for the precedence constrained knapsack problem. METEOR, Maastricht University School of Business and Economics. METEOR Research Memorandum No. 021 https://doi.org/10.26481/umamet.1997021

Document status and date:

Published: 01/01/1997

DOI:

10.26481/umamet.1997021

Document Version:

Publisher's PDF, also known as Version of record

\section{Please check the document version of this publication:}

- A submitted manuscript is the version of the article upon submission and before peer-review. There can be important differences between the submitted version and the official published version of record.

People interested in the research are advised to contact the author for the final version of the publication, or visit the DOI to the publisher's website.

- The final author version and the galley proof are versions of the publication after peer review.

- The final published version features the final layout of the paper including the volume, issue and page numbers.

Link to publication

\footnotetext{
General rights rights.

- You may freely distribute the URL identifying the publication in the public portal. please follow below link for the End User Agreement:

www.umlib.nl/taverne-license

Take down policy

If you believe that this document breaches copyright please contact us at:

repository@maastrichtuniversity.nl

providing details and we will investigate your claim.
}

Copyright and moral rights for the publications made accessible in the public portal are retained by the authors and/or other copyright owners and it is a condition of accessing publications that users recognise and abide by the legal requirements associated with these

- Users may download and print one copy of any publication from the public portal for the purpose of private study or research.

- You may not further distribute the material or use it for any profit-making activity or commercial gain

If the publication is distributed under the terms of Article $25 \mathrm{fa}$ of the Dutch Copyright Act, indicated by the "Taverne" license above, 


\title{
Lifting Valid Inequalities for the Precedence Constrained Knapsack Problem
}

\author{
R.L.M.J. van de Leensel ${ }^{1,2}$ \\ C.P.M. van Hoesel $^{1}$ \\ J.J. van de Klundert ${ }^{1}$
}

April 29, 1997

\begin{abstract}
This paper considers the precedence constrained knapsack problem. More specifically, we are interested in classes of valid inequalities which are facet-defining for the precedence constrained knapsack polytope. We study the complexity of obtaining these facets using the standard sequential lifting procedure. Applying this procedure requires solving a combinatorial problem. For valid inequalities arising from minimal induced covers, we identify a class of lifting coefficients for which this problem can be solved in polynomial time, by using a supermodular function, and for which the values of the lifting coefficients have a combinatorial interpretation. For the remaining lifting coefficients it is shown that this optimization problem is strongly NP-hard. The same lifting procedure can be applied to $(1, \mathrm{k})$-configurations, although in this case, the same combinatorial interpretation no longer applies. We also consider K-covers, to which the same procedure need not apply in general. We show that facets of the polytope can still be generated using a similar lifting technique. For tree knapsack problems, we observe that all lifting coefficients can be obtained in polynomial time. Computational experiments indicate that these facets significantly strengthen the LP-relaxation.
\end{abstract}

\section{Introduction}

The knapsack problem is a classical problem in combinatorial optimization. In this problem, there is a set $V$ of items, $V=\{1,2, \ldots, n\}$ and a knapsack with capacity $b \in \mathbb{Z}^{+}$. Each item $i \in V$ has a value $c_{i} \in \mathbb{Z}$, and a weight $w_{i} \in \mathbb{Z}^{+}$. The problem is to find a maximum value subset of the set of items whose total weight does not exceed the knapsack capacity. This is modelled in the following integer linear programming formulation:

\footnotetext{
${ }^{1}$ Dept of Quantitative Economics, Maastricht University, P.O.Box 616, 6200 MD Maastricht, The Netherlands.

${ }^{2}$ e-mail: r.vandeleensel@ke.unimaas.nl
} 


$$
\begin{array}{ll}
\max & \sum_{i=1}^{n} c_{i} x_{i} \\
\text { s.t. } & \sum_{i=1}^{n} a_{i} x_{i} \leq b \\
& x_{i} \in\{0,1\} \quad i=1, \ldots, n
\end{array}
$$

The knapsack problem has received considerable attention, not only because it has several important applications in itself, but also because it arises as a substructure in many combinatorial problems.

This paper studies the precedence constrained knapsack problem, which generalizes the knapsack problem by including a partial order on the items. We say that there is a precedence constraint from item $i$ to item $j$ if item $j$ can be included in the knapsack only if item $i$ is included. Thus, $x_{i}=0 \Rightarrow x_{j}=0$. The set of precedence constraints can be represented by a directed graph $D(V, A)$, where the node set $V$ is the set of items, and each precedence constraint is represented by a directed arc in $A$. The precedence constraints are given by

$$
x_{i} \geq x_{j} \quad(i, j) \in A
$$

The precedence constrained knapsack problem (PCKP) is now formulated by (1)-(4). In this paper, we are interested in facet-defining inequalities for the precedence constrained knapsack polytope, and more specifically, the complexity of obtaining these facets using lifting techniques. The remainder of this section discusses PCKP, the literature on its polyhedral structure, and the contributions of the present paper.

As is the case for the ordinary knapsack problem, PCKP is an interesting problem in itself, which also arises naturally as a substructure in several other combinatorial problems. Consider for instance a tool management problem that arises in automated manufacturing, where each part requires a specific set of tools in order to be processed. Hence, a part can only be processed on a machine if the required tools are loaded. In our model this would correspond to a precedence constraint from tool $i$ to job $j$ if tool $i$ is required to process job $j$. The knapsack constraint stems from the limited capacity of the tool magazine. Crama [7] and Stecke and Kim [18] discuss several problems containing both knapsack and precedence constraints in the context of tool management, and provide pointers to literature on related combinatorial problems, of which we mention only a few here. Mamer and Shogan [13] and Hwan and Shogan [11] consider capital constrained repair kit selection problems, which also have both knapsack and precedence constraints. A similar formulation arises in strip mining where digging in lower layers is impossible without digging in higher layers (see Johnson and Niemi [12]). Shaw [16] and Cho and Shaw [6] employ a decomposition technique to tackle network design problems, for which the precedence constrained knapsack problem arises as a subproblem (see Cho and Shaw [5] and Shaw and Cho [17]). 
Garey and Johnson [9] prove that the decision version of PCKP is NP-complete in the strong sense, but solvable in pseudopolynomial time if the underlying precedence graph is a tree (see Johnson and Niemi [12] for a dynamic programming algorithm). Hence, in order to solve the general PCKP to optimality, a further understanding of the structure of the precedence constrained knapsack polytope can be expected to accelerate general integer programming schemes, as it has led to more powerful exact solution methods for ordinary knapsack problems (see Crowder, Johnson and Padberg [8]). For polyhedral results on the ordinary knapsack problem we refer to Balas [2], Balas and Zemel [3] and Zemel [19]. Hartvigsen and Zemel [10] discuss the complexity of the recognition of (lifted) valid knapsack inequalities.

As is observed by Boyd [4], problems which are defined entirely by precedence constraints can be solved using standard LP-techniques, since a set of precedence constraints itself defines a totally unimodular matrix and hence, a polyhedron with integral vertices. For the PCKP, Boyd [4] analyses two classes of valid inequalities arising from K-covers and $(1, \mathrm{k})$-configurations. He identifies conditions under which these inequalities define facets of a lower dimensional polytope, in which case lifting may lead to a facet of the precedence constrained knapsack polytope itself. Park and Park [15] consider a special case of K-covers which they refer to as minimal induced covers. In general, inequalities arising from minimal induced covers will not define facets of the precedence constrained knapsack polytope. Park and Park [15] consider a lifting technique to obtain valid inequalities. The reader may observe that all of the aforementioned classes of valid inequalities are natural extensions of classes of inequalities for the ordinary knapsack problem.

In this paper, we present various new results on facets of the PCKP-polytope. In Section 3 we state a class of lifting orders which guarantees that valid inequalities of the PCKP-polytope can be lifted to obtain facet-defining inequalities for the polytope, using a standard sequential lifting procedure. For valid inequalities arising from minimal induced covers and $(1, \mathrm{k})$-configurations we identify the lower dimensional polytope for which valid inequalities arising from minimal induced covers and $(1, \mathrm{k})$-configurations are facet-defining. We specifically consider valid inequalities arising from minimal induced covers; the variables for which lifting coefficients have yet to be determined are partitioned into two classes. For one of these classes, we establish a relation between the lifting coefficients and the number of components in two related subgraphs of $D$. Based on this characterization, these lifting coefficients can be seen to be computable in polynomial time. For the second class of lifting coefficients however, we prove that their computation is strongly NP-hard. A special case of the PCKP which has received considerable attention is the tree knapsack problem, in which the underlying precedence graph is a tree. Aghezzaf, Magnanti and Wolsey [1] for instance, study the polyhedral structure of the problem. Our results allow for more general graph structures and extend their findings. Moreover, our results easily imply that, for the tree knapsack problem, all lifting coefficients can be obtained in polynomial time. In Section 4 we consider valid inequalities arising from K-covers. For these valid inequalities standard sequential lifting techniques cannot always be applied. We show that by applying a related lifting procedure facets of the PCKP-polytope can still be obtained. To illustrate the effect of lifted inequalities and their applicability in integer solution procedures, we report our computational results in Section 5. 


\section{Notation}

Throughout this paper, the following definitions and notation will turn out to be convenient. For $(i, j) \in A$, item $i$ is called a predecessor of item $j$ and item $j$ is called a successor of item $i$. For all $W \subseteq V$, we denote by $F(W)=\left\{j \in V \backslash W \mid \exists i_{1} \in W:\left(i_{1}, j\right) \in A, \exists i_{2} \in W:\left(j, i_{2}\right) \in A\right\}$ the set of elements in $V \backslash W$ which are both a successor of an element in $W$ and a predecessor of an element in $W$, by $P(W)=\{j \in V \backslash W \mid \exists i \in W:(j, i) \in A\} \backslash F(W)$ the set of predecessors of a set $W$ excluding items in $W$ and $F(W)$, by $T(W)=W \cup P(W) \cup F(W)$ the set of predecessors of set $W$ including $W$, and by $R(W)=V \backslash T(W)$ the set of remaining items (variables). For ease of exposition, $P(\{i\}), T(\{i\})$ and $R(\{i\})$ will be denoted $P(i), T(i)$ and $R(i)$, respectively. Furthermore, for all $W \subseteq V$ define $a(W)=\sum_{i \in W} a_{i}$. Note that if $(i, j) \in A$ and $(j, k) \in A$, then, by transitivity of the precendence relations, $(i, k)$ can be assumed to be an element of $A$. Arcs in $A$ induced by transitivity will be omitted in the figures. Moreover, arcs $(i, j)$ are depicted downward.

The following two assumptions can be made without loss of generality.

Assumption 1. The directed graph $D$ is acyclic.

If $D$ contains a cycle, nodes (variables) in this cycle must either all be included in, or all be excluded from the knapsack. Hence, the cycle can be contracted into a single node, with cumulative value and weight coefficient.

Assumption 2. $a(T(i)) \leq b$, for all $i=1, \ldots, n$.

This simply implies that for every item $i$ there exists a feasible solution in which it is included in the knapsack. Items violating this assumption can be deleted from the problem instance.

\section{Minimal Induced Covers and (1,k)-configurations}

In the literature on the polyhedral structure of the knapsack problem, minimal cover inequalities have been investigated (see for instance Balas [2], Balas and Zemel [3]). In order to generalize these concepts to PCKP, we must take into account that if an item $i$ is included in the knapsack, so must all the items in $T(i)$. In Subsection 3.1 we therefore consider a straightforward generalization of minimal covers, the so-called minimal induced cover (see Park and Park [15]). We show how valid inequalities for the precedence constrained knapsack polytope can be strengthened using standard sequential lifting techniques. Different lifting orders are discussed, and we derive

sufficient conditions for classes of valid inequalities under which lifting leads to facet-defining inequalities for the PCKP-polytope.

Given a minimal induced cover $C \subseteq V$, we give a combinatorial characterization of the value of the lifting coefficients for the variables in $P(C)$ in Subsection 3.2. This leads to the conclusion that these values can be computed in polynomial time. Subsection 3.3 shows that the computation of lifting coefficients for variables in $R(C)$ is, in general, strongly NP-hard, but solvable 
in polynomial time in the special case where the underlying precedence graph is a tree. Finally, Subsection 3.4 concludes this section with an illustrative example.

\subsection{Generic Sequential Lifting}

Item $i \in V$ and $j \in V$ are called incomparable if both $(i, j) \notin A$ and $(j, i) \notin A$. A set $W \subseteq V$ is called incomparable if the elements in $W$ are pairwise incomparable. Note that if $W$ is incomparable then $F(W)=\emptyset$.

Definition 3.1 $C \subseteq V$ is a minimal induced cover (MIC) if

- $C$ is incomparable

- $a(T(C))>b$

- $a(T(C) \backslash\{i\}) \leq b$, for all $i \in C$

In words, a minimal induced cover is a set of incomparable items, which together do not fit in the knapsack, whereas all but one of them do fit in the knapsack together. The above definition follows the work of Boyd [4]. An alternative definition would be to replace $a(T(C) \backslash\{i\}) \leq b$, for all $i \in C$ by $a(T(C \backslash\{i\})) \leq b$, for all $i \in C$. In fact, the latter inequality appears in the definition of minimal induced covers of Park and Park [15]. However, on close inspection it can be verified that the results of Park and Park [15] are derived under conditions for which the two definitions coincide. Since our results are only applicable under the current definition, we follow the original definition of Boyd [4].

For $C \subseteq V$ a MIC, the following inequality is valid:

$$
\sum_{i \in C} x_{i} \leq|C|-1
$$

We refer to this inequality as the $M I C$-inequality.

We define $X$ to be the set of feasible solutions of the PCKP, and $\operatorname{conv}(X)$ to be the convex hull of the set $X$. Furthermore, for any $W_{1}, W_{2} \subseteq V$ such that $W_{2} \cap T\left(W_{1}\right)=\emptyset$, we define the subset $X_{W_{1} \mid W_{2}}=X \cap\left\{x \in\{0,1\}^{n} \mid x_{i}=1\right.$, for $i \in W_{1}$, and $x_{i}=0$, for $\left.i \in W_{2}\right\}$. For $W \subseteq V$, we denote by $x^{W}$ be the characteristic vector of $W$, that is, $x_{i}^{W}=1$, if $i \in W$, and $x_{i}^{W}=0$, otherwise.

Proposition 3.1 (see Boyd [4]) The dimension of $\operatorname{conv}(X)$ is $|V|$.

Proof. The vectors $x^{T(i)}, i=1, \ldots,|V|$ together with the zero vector give $|V|+1$ affinely independent vectors in $\operatorname{conv}(X)$ (note that we use assumption 2 here). 
Proposition 3.2 Let $C \subseteq V$ be a MIC. Then (5) is facet-defining for conv $\left(X_{P(C) \mid R(C)}\right)$.

Proof. By Proposition 3.1, the dimension of conv $\left(X_{P(C) \mid R(C)}\right)$ is $|C|$. We specify $|C|$ affinely independent vectors in $\operatorname{conv}\left(X_{P(C) \mid R(C)}\right)$ satisfying (5) at equality. Let $\theta^{j}=x^{T(C) \backslash\{j\}}$, for all $j \in C$. It can easily be checked that the vectors $\theta^{j}, j \in C$ satisfy the inequality at equality and are affinely independent.

Proposition 3.2 enables us to lift the variables in $P(C)$ and $R(C)$ into the MIC-inequality using the following technique (see Nemhauser and Wolsey [14]). Let $B^{n}=\{0,1\}^{n}$. Suppose $Y \subseteq B^{n}$, $Y^{0}=Y \cap\left\{x \in B^{n} \mid x_{j}=0\right\}$, and $Y^{1}=Y \cap\left\{x \in B^{n} \mid x_{j}=1\right\}$. If the inequality

$$
\sum_{i \neq j} \alpha_{i} x_{i} \leq \alpha_{0}
$$

is facet-defining for $\operatorname{conv}\left(Y^{1}\right)$ and $Y^{0} \neq \emptyset$, then

$$
\sum_{i \neq j} \alpha_{i} x_{i}+\alpha_{j}\left(1-x_{j}\right) \leq \alpha_{0}
$$

is facet-defining for $\operatorname{conv}(Y)$ if

$$
\alpha_{j}=\alpha_{0}-\max _{x \in Y^{0}}\left\{\sum_{i \neq j} \alpha_{i} x_{i}\right\}
$$

Similarly, if (6) defines a facet for $\operatorname{con} v\left(Y^{0}\right)$ and $Y^{1} \neq \emptyset$, then

$$
\sum_{i \neq j} \alpha_{i} x_{i}+\alpha_{j} x_{j} \leq \alpha_{0}
$$

defines a facet of $\operatorname{conv}(Y)$ if

$$
\alpha_{j}=\alpha_{0}-\max _{x \in Y^{1}}\left\{\sum_{i \neq j} \alpha_{i} x_{i}\right\}
$$

In order to apply this lifting technique repeatedly to MIC-inequalities, we have to be careful with the order in which the variables are lifted. Otherwise we might, at some point, violate the condition $Y^{0} \neq \emptyset$ when applying (8) or the condition $Y^{1} \neq \emptyset$ when applying (10). 
Definition 3.2 For $W \subseteq V, \pi$ is called a PFRS-order (predecessors first, remaining variables second) for $W$ if $\pi$ is a one-to-one mapping $\pi: P(W) \cup R(W) \rightarrow\{1, \ldots,|P(W) \cup R(W)|\}$ satisfying the following conditions:
(i) $\pi(i)<\pi(j) \quad$ if $i \in P(W), j \in R(W)$
(ii) $\pi(i)<\pi(j) \quad$ if $i, j \in P(W)$ and $j \in P(i) \quad$ (reversed topological ordering on $P(W)$ )
(iii) $\pi(i)<\pi(j) \quad$ if $i, j \in R(W)$ and $i \in P(j) \quad$ (topological ordering on $R(W)$ )

Note that under assumption 1 such an order always exists. Given a MIC $C \subseteq V$ and a PFRSorder $\pi$ for $C$, for all elements $j \in P(C) \cup R(C)$ we define

$$
\begin{aligned}
& p^{\pi}(j)=\{i \in P(C) \cup R(C) \mid \pi(i)<\pi(j)\} \quad \text { (predecessors of } j \text { in order } \pi \text { ) } \\
& s^{\pi}(j)=\{i \in P(C) \cup R(C) \mid \pi(i)>\pi(j)\} \quad \text { (successors of } j \text { in order } \pi \text { ) }
\end{aligned}
$$

During the lifting process, variables in $P(C) \cup R(C)$ are lifted sequentially, and hence, the lifting problem for a variable $j \in P(C)$ is defined as follows:

Given a MIC $C \subseteq V$ and its associated MIC inequality

$$
\sum_{i \in C} x_{i}+\sum_{i \in P(C) \cap p^{\pi}(j)} \alpha_{i}\left(1-x_{i}\right) \leq|C|-1
$$

which is valid for conv $(X)$, determine

$$
\alpha_{j}=|C|-1-\max _{\substack{x \in X_{P(C) \cap s^{\pi}(j) \mid R(C)} \\ x_{j}=0}}\left\{\sum_{i \in C} x_{i}+\sum_{i \in P(C) \cap p^{\pi}(j)} \alpha_{i}\left(1-x_{i}\right)\right\} .
$$

Likewise, for a variable $j \in R(C)$ the lifting problem is defined as follows:

Given a MIC $C \subseteq V$ and an inequality

$$
\sum_{i \in C} x_{i}+\sum_{i \in P(C)} \alpha_{i}\left(1-x_{i}\right)+\sum_{i \in R(C) \cap p^{\pi}(j)} \alpha_{i} x_{i} \leq|C|-1
$$

which is valid for $X$, determine

$$
\alpha_{j}=|C|-1-\max _{\substack{x \in X_{\emptyset \mid R(C) \cap s^{\pi}(j)} \\ x_{j}=1}}\left\{\sum_{i \in C} x_{i}+\sum_{i \in P(C)} \alpha_{i}\left(1-x_{i}\right)+\sum_{i \in R(C) \cap p^{\pi}(j)} \alpha_{i} x_{i}\right\}
$$


Theorem 3.1 Let

- $C \subseteq V$ be a MIC with its corresponding valid inequality (5)

- $\pi$ be a PFRS-order for $C$.

- lifting coefficients for variables in $P(C)$ be determined according to (12)

- lifting coefficients for variables in $R(C)$ be determined according to (14)

then the resulting inequality

$$
\sum_{i \in C} x_{i}+\sum_{i \in P(C)} \alpha_{i}\left(1-x_{i}\right)+\sum_{i \in R(C)} \alpha_{i} x_{i} \leq|C|-1
$$

defines a facet of the PCKP-polytope conv $(X)$.

Proof. We construct $|V|$ affinely independent vectors in $\operatorname{conv}(X)$, satisfying the inequality at equality. For $j \in C$, let $\theta^{j}$ be defined as in the proof of Proposition 3.2. For all $j \in P(C)$, let $\theta^{j}$ be the vector for which the maximum in (12) is attained. W.l.o.g. assume that $\theta_{i}^{j}=1$ for all $i \in P(C) \cap s^{\pi}(j)$, and $\theta_{i}^{j}=0$, for all $i \in R(C)$. Likewise, for all $j \in R(C)$, let $\theta^{j}$ be the vector for which the maximum in (14) is attained. W.l.o.g., assume that $\theta_{i}^{j}=0$, for all $i \in R(C) \cap s^{\pi}(j)$. Then it is easy to verify that the vectors $\theta^{j}, j \in V$, satisfy the inequality at equality, and moreover, are affinely independent.

In order for the lifting procedure that consists of repeatedly solving (12) and (14) to be applicable, the maximum that is taken in (12) and (14) has to be well defined. This is not the case, if the subset over which the maximum is taken is empty. We conclude that the procedure is only valid if, at each iteration, the subset is nonempty. In the current framework, of lifting MIC inequalities, this property is ascertained by the ordering conditions stated in Definition (3.2). This result, of course, can be generalized to wider classes of valid inequalities, which may even be defined on subsets which need not necessarily be incomparable.

Theorem 3.2 Let $W \subseteq V$, and let $\alpha^{T} x \leq \alpha_{0}$, where $\alpha_{j}=0$ for $j \notin W$, be a facet-defining inequality for conv $\left(X_{P(W) \mid R(W)}\right)$. Then, lifting the variables in $P(W)$ and $R(W)$ as specified in (8) and (10) in PFRS-order yields a facet of the PCKP-polytope conv $(X)$.

Proof. Let $W \subseteq V$. If $W=\emptyset$, then $X_{P(W) \mid R(W)}=X$, no lifting has to be done, and hence the theorem obviously holds. So assume $W \neq \emptyset$. As we are given a facet-defining inequality, $X_{P(W) \mid R(W)} \neq \emptyset$, which implies that the vector $x^{P(W)} \in X$ (i.e. $a(P(W)) \leq b$ ). Now it only remains to proof that at every step of the lifting process the subset on which the maximum in (8) and (10) is defined is nonempty. Under a PFRS-order $\pi$, when lifting $j \in P(W)$, since 
$a\left(P(W) \cap s^{\pi}(j)\right) \leq a(P(W)) \leq b$, the vector $x^{P(W) \cap s^{\pi}(j)}$ is in the corresponding subset. When lifting $j \in R(W)$, the vector $x^{T(j)}$ is in the subset at hand.

The conditions on the PFRS-order in Definition 3.2 are such that at each step of the lifting process the variables which are fixed do not violate the precedence constraints, and the variables which are fixed to one do not violate the knapsack constraint. Instead of considering a PFRSorder in which all elements in $P(W)$ are lifted before elements in $R(W)$, we might also allow for more general lifting orders, in which an element in $R(W)$ can be lifted before all predecessors in $P(W)$ are lifted. The existence of such an order is again guaranteed by assumption 1 . Next, we derive necessary and sufficient conditions for which this class of more general orders yields facet-defining inequalities.

Definition 3.3 For $W \subseteq V, \pi$ is called a valid order for $W$ if $\pi$ is a one-to-one mapping $\pi: P(W) \cup R(W) \rightarrow\{1, \ldots,|P(W) \cup R(W)|\}$ satisfying the following conditions:
(i) $\pi(i)<\pi(j) \quad$ if $i, j \in P(W) \backslash W$ and $j \in P(i)$
(reversed topological ordering on $P(W)$ )
(ii) $\pi(i)<\pi(j) \quad$ if $i, j \in R(W) \backslash W$ and $i \in P(j)$
(topological ordering on $R(W)$ )

Theorem 3.3 Let $W \subseteq V$, and let $\alpha^{T} x \leq \alpha_{0}$, where $\alpha_{j}=0$ for $j \notin W$, be a facet-defining inequality for conv $\left(X_{P(W) \mid R(W)}\right)$. Let $\pi$ be a valid order for $W$. Let the lifting coefficients of the variables in $P(W)$ and $R(W)$ be determined as in (8) and (10). Then the resulting inequality is facet-defining for conv $(X)$ if and only if $a\left(\left(P(W) \cap s_{j}^{\pi}\right) \cup T(j)\right) \leq b$, for each $j \in R(W)$.

Proof. Let $W \subseteq V$. If $W=\emptyset$, then $X_{P(W) \mid R(W)}=X$, no lifting has to be done, and hence the theorem obviously holds. So assume $W \neq \emptyset$. Using inductive arguments, when lifting a variable $x_{j}$ we are given a facet-defining inequality for the polytope $\operatorname{conv}\left(X_{P(W) \cap s_{j}^{\pi} \mid R(W) \cap s_{j}^{\pi}}\right)$. If $j \in P(W)$ then by nonemptyness of the above polytope and the definition of a valid order, the subset on which the maximum as in (8) is defined is nonempty since the vector $x^{P(W) \cap s^{\pi}(j)}$ is in the corresponding polytope. If $j \in R(W)$, then the condition on the weights as mentioned in the theorem guarantees that the subset on which the maximum is defined as in (10) is nonempty since the vector $x^{\left(P(W) \cap s^{\pi}(j)\right) \cup T(j)}$ is in the corresponding polytope. Conversely, assume the condition is not satisfied for a $j \in R(W)$. When lifting the corresponding variable, the subset on which the maximum is defined is empty.

It is easy to see that conditions $(i)$ and $(i i)$ in Definition 3.3 cannot be removed since then immediately the subset on which the maximum is defined in the lifting procedure will become empty. Hence the class of valid orders in Definition 3.3 is the most general class of orders which can be considered for the standard lifting procedure as defined by (8) and (10). Note that the above result paves the way for lifting other classes of valid inequalities. Here, we mention $(1, \mathrm{k})$-configurations and state the polytope for which the corresponding valid inequality is facetdefining. 


\section{Definition 3.4 Let}

- $C \cup\{t\} \subseteq V$ be incomparable, with $t \notin C$

- $C \cup\{t\}$ be a cover and $a(T(C \cup\{t\}) \backslash\{t\}) \leq b$

- $Q \cup\{t\}$ is a minimal (induced) cover, $\forall Q \subseteq C$ with $|Q|=k$ where $2 \leq k \leq|C|$

then $C \cup\{t\}$ is called a $(1, k)$-configuration.

For a $(1, \mathrm{k})$-configuration the following set of inequalities are valid:

$$
(r-k+1) x_{t}+\sum_{i \in Z(r)} x_{i} \leq r
$$

where $r$ is such that $k \leq r \leq|C|$ and $Z(r)$ is any subset of $C$, with $|Z(r)|=r$.

Proposition 3.3 Let $C \cup\{t\} \subseteq V$ be a $(1, k)$-configuration, and let $Z(r)$ be any subset of $C$, with $|Z(r)|=r$. Then, (16) is facet-defining for conv $\left(X_{P(C \cup\{t\}) \mid R(C \cup\{t\}) \cup(C \backslash Z(r))}\right)$.

Proof. It follows from Proposition 3.1 that the dimension of $\operatorname{conv}\left(X_{P(C \cup\{t\}) \mid R(C \cup\{t\}) \cup(C \backslash Z(r))}\right)$ is $r+1$. Hence, to prove the proposition, we construct $r+1$ affinely independent vectors in the polytope satisfying the inequality at equality. We assume without loss of generality that the elements are numbered such that $1, \ldots, r$ denote the elements in $Z(r)$ and $r+1$ denotes element $t$.

For $i=1, \ldots, r-k+2$, let $y^{i}$ be defined by

$$
y_{j}^{i}= \begin{cases}1 & j \in\{i, \ldots, i+k-2\} \\ 0 & j \in\{1, \ldots, r\} \backslash\{i, \ldots, i+k-2\} \\ 1 & j=r+1 \\ 1 & j \in P(C \cup\{t\}) \\ 0 & j \in R(C \cup\{t\}) \cup(C \backslash Z(r))\end{cases}
$$

For $i=r-k+3, \ldots, r$, let $y^{i}$ be defined by

$$
y_{j}^{i}= \begin{cases}1 & j \in\{i, \ldots, r\} \cup\{1, \ldots, i+k-r-2\} \\ 0 & j \in\{i+k-r-1, \ldots, i-1\} \\ 1 & j=r+1 \\ 1 & j \in P(C \cup\{t\}) \\ 0 & j \in R(C \cup\{t\}) \cup(C \backslash Z(r))\end{cases}
$$


and define $y^{r+1}$ as

$$
y^{r+1}= \begin{cases}0 & j=r+1 \\ 1 & j \in Z(r) \\ 1 & j \in P(C \cup\{t\}) \\ 0 & j \in R(C \cup\{t\}) \cup(C \backslash Z(r))\end{cases}
$$

Then it can easily be verified that the vectors $y^{i}, i=1, \ldots, r+1$ are affinely independent.

Applying Theorem 3.2 or 3.3 now yields that the elements of $V \backslash(Z(r) \cup\{t\})$ can now be lifted in PFRS-order or valid order so as to obtain a facet of $\operatorname{conv}(X)$.

\subsection{Lifting Predecessors of a Minimal Induced Cover using a PFRS-order}

In general, calculating $\alpha_{j}$ by (8) or (10) requires solving a difficult maximization problem. In fact, for PCKP, the optimization problems in (12) and (14) are in turn PCKP problems. In this Subsection we show that the lifting problem of predecessors under a PFRS-order has a combinatorial interpretation that leads to an algorithm that solves the lifting problem in polynomial time. In contrast, in Subsection 3.3, it is shown that the lifting problem for the remaining variables is strongly NP-hard.

Definition 3.5 Let $C \subseteq V$ be a MIC. For $W \subseteq P(C)$ let $f(W)$ be the number of (weak) components in the subgraph of $G$ induced by $W \cup C$.

Lemma $3.1 f$ is supermodular: for all $W_{1} \subseteq W_{2} \subseteq P(C)$ and $i \in P(C) \backslash W_{2}$ it holds that

$$
f\left(W_{1} \cup\{i\}\right)-f\left(W_{1}\right) \leq f\left(W_{2} \cup\{i\}\right)-f\left(W_{2}\right)
$$

Proof. Choose $W_{2} \subseteq P(C)$ and $W_{1} \subseteq W_{2}$ arbitrarily. Notice first that, since we only consider $W_{1} \subseteq P(C)$ and $W_{2} \subseteq P(C)$, each component of a subgraph induced by $W_{1} \cup C$ or $W_{2} \cup C$ contains at least one element $c \in C$. Further, since $W_{1} \subseteq W_{2}$, it must hold that if $c_{1} \in C$ and $c_{2} \in C$ are in different components of the subgraph induced by $W_{2} \cup C$, then they also are in different components of the subgraph induced by $W_{1} \cup C$.

Now, consider a component of $W_{2} \cup C \cup\{i\}$, containing vertices $Q$, and let $K=Q \backslash C$. Since this component contains at least one element of $C$, the subgraph induced by $Q \backslash\{i\}$, consists of a strictly positive number of say $k$ components. Let $K_{j}, j=1, \ldots, k$ be the nodes in the intersection of $W_{2}$ and the $j$-th of these components (the components may be numbered arbitrarily), and similarly, let $C_{j}, j=1, \ldots, k$ be the nodes in the intersection of $C$ and the $j$-th of these components. The subgraphs induced by $K_{j} \cup C_{j}, j=1, \ldots, k$ form the distinct components of $W_{2} \cup C$. 
We first consider the case where $i \in Q$. Since for all $j=1, \ldots, k$, the subgraphs induced by $K_{j} \cup C_{j}$ contain at least one element of $C$, and since they are distinct components of the subgraph induced by $W_{2} \cup C$, there must be distinct $c_{j} \in C_{j}, j=1, \ldots, k$ such that $i \in P\left(c_{j}\right), j=1, \ldots, k$. Using that $W_{1} \subseteq W_{2}$, we establish that all $c_{j}, j=1, \ldots, k$ are in distinct components of the subgraph induced by $W_{1} \cup C$. Let $K_{j}^{\prime}$ be the set of elements of $P(C)$ that are in the component containing $c_{j}$ in the subgraph induced by $W_{1} \cup C$. Then, $K_{j}^{\prime} \subseteq K_{j}$, since $W_{1} \subseteq W_{2}$. Further, since $i \in P\left(c_{j}\right), j=1, \ldots, k$, these $k$ components $K_{j}^{\prime}$ are in a single component of the subgraph induced by $W_{1} \cup C \cup\{i\}$. Let $K^{\prime}$ be the intersection of the set of nodes in this component and the nodes in $P(C)$, and let $C^{\prime}$ be the other nodes in the component. Hence, the component $Q$ of the subgraph induced by $\left(W_{2} \cup C \cup\{i\}\right)$ containing $i$, which consists of $k$ distinct components $K_{1} \cup C_{1}, \ldots, K_{k} \cup C_{k}$ of the subgraph induced by $W_{2} \cup C$, contains as a subgraph a component $K^{\prime} \cup C^{\prime}$ of the subgraph induced by $W_{1} \cup C \cup\{i\}$ that contains in turn at least $k$ components $K_{1}^{\prime} \cup C_{1}^{\prime}, \ldots, K_{k}^{\prime} \cup K_{k}^{\prime}$ of the subgraph induced by $W_{1} \cup C$, such that $K_{j}^{\prime} \subseteq K_{j}$ for $j=1, \ldots, k$. Thus it holds that

$$
\left.f\left(W_{2} \cap Q\right)-f\left(\left(W_{2} \cap Q\right) \cup\{i\}\right) \geq f\left(W_{1} \cap Q\right)-f\left(W_{1} \cap Q\right) \cup\{i\}\right)
$$

On the other hand, if $i \notin Q, Q \backslash\{i\}=Q$, and hence the subgraph induced by $Q \backslash\{i\}$ consists of one component of the subgraph induced by $W_{2} \cup C$, namely the component induced by the vertices in $Q$. Now consider the set $Q \cap C$, and observe that $i$ is not a predecessor of any of the elements in $Q \cap C$. Consider a component $Q^{\prime}$ in $W_{1} \cup C$ for which it holds that $Q^{\prime} \subseteq Q$. Since $i$ is not a predecessor of any vertex in $Q$, it is not a predecessor of any element of $Q^{\prime}$, and thus, $Q^{\prime}$ is a component of $W_{1} \cup C \cup\{i\}$. Hence, in this case we have that

$$
0=f\left(W_{2} \cap Q\right)-f\left(\left(W_{2} \cap Q\right) \cup\{i\}\right)=f\left(W_{1} \cap Q\right)-f\left(\left(W_{1} \cap Q\right) \cup\{i\}\right)
$$

Since each component of $W_{1} \cup\{i\}$ is contained in a component of $W_{2} \cup\{i\}$, and since the components of $W_{2} \cup\{i\}$ are, by definition, disjoint, this implies that

$$
\left.f\left(W_{2}\right)-f\left(W_{2} \cup\{i\}\right) \geq f\left(W_{1}\right)-f\left(W_{1}\right) \cup\{i\}\right)
$$

as required.

Definition 3.6 Let $C \subseteq V$ be a MIC and let $P(C)=\{1, \ldots, m\}$. Then, for $i \in P(C)$, let $\gamma_{i}$ be defined by

$$
\gamma_{i}=f(\{1, \ldots, i-1\})-f(\{1, \ldots, i\})
$$

Thus, $\gamma_{i}$ represents the reduction in the number of components by adding node $i$ and the arcs constituting the precedence relations in which $i$ is involved to the subgraph of $G$ induced by $C \cup\{1, \ldots, i-1\}$. As node $i$ is in $P(C)$, this automatically implies that $\gamma_{i} \geq 0$. 
Proposition 3.4 Let $C \subseteq V$ be a $M I C$ and $W \subseteq P(C)$ with $W=\{1, \ldots, m\}$. Consider the subgraph of $G$ induced by $W \cup C$. For each component $K$ of this subgraph it holds that

$$
\sum_{i \in K \cap W} \gamma_{i}=|C \cap K|-1
$$

Proof. We use induction on the elements in $W$. For $i=1$, consider any component $K$ of the subgraph induced by $C \cup\{i\}$. If component $K$ does not contain node $i$, the result follows immediately, as both the summation of the coefficients $\gamma_{i}$ in $K$ and $|C \cap K|-1$ equal zero. If component $K$ does contain node $i$, this node is connected with $|C \cap K|$ nodes in the component $K$. Hence, $|C \cap K|$ components have been merged into one component, such that the reduction in the number of components $\gamma_{i}$ equals $|C \cap K|-1$.

Next assume that the result holds for the graph induced by $C \cup\{1, \ldots, i-1\}$. Let $K$ be any component of the graph induced by $C \cup\{1, \ldots, i\}$. If $K$ does not contain node $i$, the result follows directly from the induction hypothesis. If $K$ does contain node $i$, then node $i$ merges a number of components of the graph induced by $C \cup\{1, \ldots, i-1\}$ together, say components $K_{1}, \ldots, K_{k}$. Note that this implies $\gamma_{i}=k-1$. Consequently,

$$
\sum_{i \in K \cap\{1, \ldots, i\}} \gamma_{i}=\left|C \cap K_{1}\right|-1+\ldots+\left|C \cap K_{k}\right|-1+k-1=|C \cap K|-1
$$

In the sequel we will use a special ordering on the elements from $P(C)$, namely, a reversed topological ordering, i.e. a one-to-one mapping $\pi: P(C) \rightarrow\{1, \ldots,|P(C)|\}$ satisfying $\pi(i)<\pi(j)$ for $i, j \in P(C)$ and $j \in P(i)$. The following theorem shows that under a reversed topological ordering $\gamma_{j}$ is exactly the lifting coefficient $\alpha_{j}$, as defined in (12), for $j \in P(C)$.

Theorem 3.4 Let $C \subseteq V$ be a MIC and let $\pi$ be a reversed topological ordering on $P(C)$. If the lifting coefficients $\gamma_{i}$ are determined according to (17) under the order $\pi$, then for each $j=\pi^{-1}(1), \ldots, \pi^{-1}(|P(C)|)$ the inequality

$$
\sum_{i \in C} x_{i}+\sum_{i=\pi^{-1}(1)}^{\pi^{-1}(j)} \gamma_{i}\left(1-x_{i}\right) \leq|C|-1
$$

is valid and facet-defining for the polytope conv $\left(X_{A \mid R(C)}\right)$, where $A=\left\{\pi^{-1}(j+1), \ldots, \pi^{-1}(|P(C)|)\right\}$.

Proof. To show validity of (18) it suffices to show this for $j=\pi^{-1}(|P(C)|)$. For other values of $j$ validity then follows from the fact that we restrict the set of feasible solutions by setting the variables in $\left\{\pi^{-1}(j+1), \ldots, \pi^{-1}(|P(C)|)\right\}$ to 1 . Let $x$ be an arbitrary feasible solution with 
$x_{i}=0$ for all $i \in R(C)$. Define $C^{\prime}=\left\{i \in C \mid x_{i}=1\right\}$, and $W=\left\{i \in P(C) \mid x_{i}=0\right\}$. Then obviously $C^{\prime} \neq C$ since $C$ is a MIC: $\left|C^{\prime}\right| \leq|C|-1<|C|$. Hence,

$$
\sum_{i \in C} x_{i}+\sum_{i=\pi^{-1}(1)}^{\pi^{-1}(|P(C)|)} \gamma_{i}\left(1-x_{i}\right)=\left|C^{\prime}\right|+\sum_{i \in W} \gamma_{i} \leq\left|C^{\prime}\right|+\sum_{i \in W} \gamma_{i}^{\prime} \leq\left|C^{\prime}\right|+|C|-f(W) \leq|C|-1
$$

The first inequality follows from the supermodularity of $f$, where the $\gamma_{i}$ are obtained using the sequence of $P(C)=\pi^{-1}(1), \ldots, \pi^{-1}(|P(C)|)$ and the $\gamma_{i}^{\prime}$ are obtained using the subsequence of $\pi^{-1}(1), \ldots, \pi^{-1}(|P(C)|)$ defined by $W$. Using (17), observe that $\sum_{i \in W} \gamma_{i}^{\prime}$ equals $f(\emptyset)-f(W)=$ $|C|-f(W)$. Finally, $f(W)$ equals the number of components in the graph induced by $C \cup W$. If $W=\emptyset$, then $f(W)=|C|$, and the result follows from $C^{\prime} \subset C$ since $C$ is a MIC. If $W \neq \emptyset$, it follows from solution vector $x$ that nodes in $C^{\prime}$ are no successors of nodes in $W$. Hence, in the graph induced by $C \cup W$ the nodes in $C^{\prime}$ are $\left|C^{\prime}\right|$ individual components. As there is at least one component containing elements in $W$, it follows that $f(W) \geq\left|C^{\prime}\right|+1$, which completes the proof of the validity of (18).

To show that (18) is facet-defining, we use induction on the elements $j=\pi^{-1}(1), \ldots, \pi^{-1}(|P(C)|)$. It suffices to display a feasible solution $\theta^{j}$ with $\theta_{j}^{j}=0, \theta_{i}^{j}=1, i \in\left\{\pi^{-1}(j+1), \ldots, \pi^{-1}(|P(C)|)\right\}$, and $\theta_{i}^{j}=0$ for $i \in R(C)$, satisfying (18) at equality (see Nemhauser and Wolsey [14] proposition 1.1., page 261). To construct this vector consider the graph induced by $C \cup\left\{\pi^{-1}(1), \ldots, \pi^{-1}(j)\right\}$. Let $K$ be the component containing node $j$. Define the vector $\theta^{j}$ as follows:

$$
\theta_{i}^{j}= \begin{cases}1 & i \in T(C) \backslash K \\ 0 & \text { otherwise }\end{cases}
$$

To show that $\theta^{j}$ satisfies (18) at equality, note that

$$
\begin{aligned}
\sum_{i \in C} \theta_{i}^{j}+\sum_{i=\pi^{-1}(1)}^{\pi^{-1}(j)} \gamma_{i}\left(1-\theta_{i}^{j}\right) & =|C \backslash K|+\sum_{i \in P(C) \cap K} \gamma_{i} \\
& =|C \backslash K|+|C \cap K|-1 \\
& =|C|-1
\end{aligned}
$$

where the second equality follows from Proposition 3.4.

Hence, for $j=\pi^{-1}(1)$ the vector $\theta^{j}$ satisfies the requirements. Next assume that the result holds for all $i \in\left\{\pi^{-1}(1), \ldots, \pi^{-1}(j-1)\right\}$. Hence, we are given $C+j-1$ affinely independent vectors which all have their $i^{t h}$ component equal to 1 for $i \in P(C) \backslash\left\{\pi^{-1}(1), \ldots, \pi^{-1}(j-1)\right\}$. By defining $\theta^{j}$ as in the above and following the same reasoning, $\theta^{j}$ satisfies (18) at equality and is affinely independent from the foregoing vectors, as $\theta_{j}^{j}=0$. 
Theorem 3.4 shows that $f$ can be used in a lifting process to obtain facet-defining inequalities. Clearly, given the interpretation of the coefficients in the above, their values can be determined in polynomial time, using a simple algorithm for counting components.

\subsection{Lifting of Non-Predecessor Variables of a Minimal Induced Cover}

Although the maximization problem in (8) is NP-hard in general, for PCKP the resulting problem in (12) can be solved in polynomial time when lifting predecesoors of a MIC $C$. For variables in $R(C)$, the maximization problem in equality (14) is also essentially a PCKP, but will turn out to be NP-hard in general. To give a formal proof of this statement, we introduce the following problem definitions:

Clique (see Garey and Johnson [9])

INSTANCE: Graph $G=(U, E)$, and a positive integer $K$, with $3 \leq K \leq|U|$.

QUESTION: Does $G$ contain a clique of size $K$ or more?

Note that the assumption $K \geq 3$ does not change the complexity of the problem.

\section{PCKP-MIC-R(C)-lifting}

INSTANCE:

- Instance $I$ of $P C K P$, consisting of a directed acyclic graph $D^{I}=\left(V^{I}, A^{I}\right)$, a knapsack capacity $B^{I} \in \mathbb{Z}^{+}$, and for all $i \in V^{I}$ a value $c_{i}^{I} \in \mathbb{Z}$ and a weight $a_{i}^{I} \in \mathbb{Z}^{+}$.

- A MIC $C^{I} \subseteq V^{I}$

- A PFRS-order $\pi^{I}$ on $V^{I} \backslash C^{I}$

- A facet-defining inequality

$$
\sum_{i \in C^{I}} x_{i}+\sum_{i \in P\left(C^{I}\right)} \alpha_{i}\left(1-x_{i}\right) \leq\left|C^{I}\right|-1
$$

obtained by applying the lifting procedure as defined in (12) to the variables in $P\left(C^{I}\right)$ under PFRS-order $\pi^{I}$.

- $\alpha^{I} \in \mathbb{Z}$

QUESTION: Is the lifting coefficient for the first variable in $R\left(C^{I}\right)$ under order $\pi^{I}$, as defined in (14), less than or equal to $\alpha^{I}$ ?

Theorem 3.5 PCKP-MIC-R(C)-lifting is NP-complete in the strong sense. 
Proof. It can easily be checked that PCKP-MIC-R(C)-lifting is in NP. Hence it suffices to show that Clique reduces to PCKP-MIC-R(C)-lifting. To this purpose, the graph $G=(U, E)$ will be transformed into an instance $D^{I}=\left(V^{I}, A^{I}\right)$, in which there is a node for each $u \in U$ and for each $e \in E$. The nodes corresponding to $U$ will function as predecessors of nodes corresponding to $E$. As shown in Subsection 3.2, a lifting coefficient of a predecessor is equal to the reduction in the number of components. Under the assumption that, for all $u \in U$, the degree $|\delta(u)| \geq 2$, this reduction, and hence the lifting coefficients, are enforced to be 1 . Since we are looking for cliques of size at least 3 , this assumption causes no loss of generality.

Let $G=(U, E)$ be an instance of Clique, let $K$ be any integer satisfying $3 \leq K \leq|U|$, and let $\pi$ be any order on the nodes in $U$, i.e., $U=\left\{\pi^{-1}(1), \ldots, \pi^{-1}(n)\right\}$. We define an index set $J$, consisting of nodes $u \in U$ which are currently not adjacent to a node with higher index. Hence,

$$
J=\{u \in U \mid \nexists w \in U: \pi(w)>\pi(u),\{u, w\} \in E\}
$$

Extend the graph $G$ to $\tilde{G}=(\tilde{U}, \tilde{E})$, where

$$
\tilde{U}=U \cup \bigcup_{u \in J}\{\tilde{u}\} \quad ; \quad \tilde{E}=E \cup \bigcup_{u \in J}\{u, \tilde{u}\}
$$

Furthermore, let $\tilde{K}=K$, and $\tilde{\pi}$ an order on $\tilde{U}$ such that $\pi(u)=\tilde{\pi}(u)$, for $u \in U$. Then, since nodes in $\tilde{U}$ have degree $1, G$ contains a clique of size $K \geq 3$ if and only if $\tilde{G}$ contains a clique of size $\tilde{K}$.

Next, we determine a subset $L \subseteq E$ whose elements share a common predecessor in the directed graph to be introduced shortly. Let $\delta(u)$ be the set of edges incident to node $u$ in graph $\tilde{G}$. Then, the set $L$ is determined by the following algorithm, which is to be explained shortly:

$$
\begin{aligned}
& L=\emptyset ; W=\tilde{E} ; \quad \quad \quad{ }^{*} \text { initialisation */ } \\
& \text { for } u=\tilde{\pi}^{-1}(1) \text { to } \tilde{\pi}^{-1}(|U|) \text { do } \\
& \text { begin } \\
& \text { let } e \in W \cap \delta(u) \text {; } \\
& L=L \cup(W \cap \delta(u) \backslash\{e\}) ; \\
& W=W \backslash \delta(u) ; \\
& \text { end; } \\
& \text { endfor; }
\end{aligned}
$$

This algorithm processes the vertices $u \in \tilde{U}$ in increasing order of their indices, and considers the intersection of $\delta(u)$ and $W$, where $W$ initially consist of all edges in $\tilde{E}$. In each iteration, the algorithm selects an arbitrary edge $e$ in the intersection of $\delta(u)$ and $W$, and eliminates all 
edges in $\delta(u)$ from $W$. Except for $e$, the thus eliminated edges are added to a set $L$ which is initially empty. Notice that such an $e$ always exists since each vertex $u \in U$ is adjacent to a higher index vertex in $\tilde{U}$. Notice also that all edges in $W \cap \delta(u) \backslash\{e\}$ are in $E$ since vertices in $U$ only contain edges to $\tilde{U}$, and hence in $\tilde{E} \backslash E$, if they are not adjacent to a higher index vertex in $U$, and should this be the case, then they contain only one such edge (which per force is chosen to be $e$ ).

Now, we are able to define the instance $I$ with directed graph $D^{I}=\left(V^{I}, A^{I}\right)$ where

$$
V^{I}=\tilde{U} \cup \tilde{E} \cup\{Q, q\} \quad ; \quad A^{I}=\{(u, e) \mid u \in \tilde{U}, e \in \tilde{E}, e \in \delta(u)\} \cup(Q, q) \cup \bigcup_{e \in L}(Q, e)
$$

To complete the instance $I$ of PCKP-MIC-R(C)-lifting, let $c_{v} \in \mathbb{Z}$ for all $v \in V^{I}$, and $a(Q) \in \mathbb{Z}^{+}$. Further, let

$$
B^{I}=\frac{|U|}{\tilde{K}}+|\tilde{U} \backslash U| \cdot\left[1+\frac{\left(\begin{array}{c}
\tilde{K} \\
2
\end{array}\right)}{\tilde{K}^{3}}\right]+\frac{|\tilde{E}|-1}{\tilde{K}^{3}}+a(Q)
$$

and define weights for nodes in $V^{I}$ as follows:

$$
a_{u}^{I}= \begin{cases}\frac{1}{\tilde{K}} & u \in U \\ 1+\frac{(\tilde{K})}{\tilde{K}^{3}} & u \in \tilde{U} \backslash U \\ \frac{1}{\tilde{K}^{3}} & u \in \tilde{E} \\ a(Q) & u=Q \\ B^{I}-\left[1+\frac{\left(\frac{\tilde{K}}{2}\right)}{\tilde{K}^{3}}+a(Q)\right] & u=q\end{cases}
$$

Now consider $\tilde{E}$. In order to include all $e \in \tilde{E}$ in the knapsack, so must be all items in $P(\tilde{E})$, which means in this case that all $u \in \tilde{U}$ and $Q$ must be in the knapsack. Since the nodes in $u \in \tilde{U}$ and $Q$ already account for a weight of

$$
\frac{|U|}{\tilde{K}}+|\tilde{U} \backslash U| \cdot\left[1+\frac{\left(\begin{array}{c}
\tilde{K} \\
2
\end{array}\right)}{\tilde{K}^{3}}\right]+a(Q)
$$

only $|\tilde{E}|-1$ elements from $\tilde{E}$ can be included in the knapsack. This yields that $\tilde{E}$ is a MIC. Now, let $\pi^{I}$ be a PFRS-order on $\tilde{U} \cup\{Q, q\}$ defined by:

$$
\pi^{I}(i)= \begin{cases}1 & i=Q \\ \tilde{\pi}(i)+1 & i \in \tilde{U} \\ |\tilde{U}|+2 & i=q\end{cases}
$$


and the corresponding facet-defining inequality after lifting $Q$ and $\tilde{U}$ be given by

$$
\sum_{i \in \tilde{E}} x_{i}+(|L|-1)\left(1-x_{Q}\right)+\sum_{i \in U}\left(1-x_{i}\right) \leq|\tilde{E}|-1
$$

The lifting coefficients can be explained as follows. Notice that by the construction of $A^{I}, Q$ connects $|L|$ elements of $\tilde{E}$, and thus adding $Q$ yields a reduction in the number of components of $|L|-1$. Further, by the construction of $D^{I}$, there are $|U|$ edges not connected to $Q$. Now, using our assumption all vertices $u \in U$ have $|\delta(u)| \geq 2$, each vertex $u \in U$ connects the edge $e$ selected in the $\tilde{\pi}(u)$ - th iteration of the previously described algorithm to the component which became connected when $x_{Q}$ was lifted. Hence $\alpha_{u}=1$ for all $u \in U$. Finally, the vertices $\tilde{u} \in \tilde{U}$ can be seen not to cause a reduction in the number of components at all, and hence have coefficient $\alpha_{\tilde{u}}=0$.

Finally, let

$$
\alpha^{I}=|\tilde{E}|-1-\left[\left(\begin{array}{c}
\tilde{K} \\
2
\end{array}\right)+|U|-\tilde{K}\right.
$$

We leave it to the reader to verify that the above transformation from $G$, via $\tilde{G}$, to instance $I$ is polynomial.

Next let us consider the problem (14) that arises when lifting $x_{q}$, the only variable in $R\left(C^{I}\right)$. When lifting variable $x_{q}$ according to (14), nodes $q$ and $Q$ must be included in the knapsack.

We are now going to show that $\tilde{G}$ contains a clique of size $\tilde{K} \geq 3$ if and only if the maximal value of the lifting coefficient for variable $x_{q}$ is less than or equal to $\alpha^{I}$.

$(\Rightarrow)$ If $G$ contains a clique of size $\tilde{K}$, then by including the nodes $u$ in $\tilde{U} \cup \tilde{E}$ corresponding to the vertices and edges in the clique in the knapsack together with $Q$ and $q$, we have a cumulative weight $\frac{\tilde{K}}{\tilde{K}}+\frac{(\tilde{K})}{\tilde{K}^{3}}+a(Q)+B^{I}-\left[1+\frac{\left(\frac{\tilde{K}}{2}\right)}{\tilde{K}^{3}}+a(Q)\right]=B^{I}$. Thus this set of items is feasible. Furthermore, together these items yield a value for $\alpha^{I}$ of

$$
|\tilde{E}|-1-\left[\left(\begin{array}{c}
\tilde{K} \\
2
\end{array}\right)+|U|-\tilde{K}\right]
$$

as required.

$(\Leftarrow)$ Let the value of the lifting coefficient for $x_{q}$ (being $\alpha_{q} \leq \alpha^{I}$ ) be obtained by a solution in which $i$ items (say) corresponding to nodes $u \in U$ in the graph $\tilde{G}$ are included in the knapsack. 
Let us first assume $i>\tilde{K}$. Then the weight in the knapsack amounts to at least

$$
1+\frac{1}{\tilde{K}}+a(Q)+B^{I}-\left[1+\frac{\left(\begin{array}{c}
\tilde{K} \\
2
\end{array}\right)}{\tilde{K}^{3}}+a(Q)\right]>B^{I}
$$

which yields a contradiction. Hence $i \leq \tilde{K}$. Assume $i<\tilde{K}$. Then,

$$
\alpha_{q} \geq|\tilde{E}|-1-\left[\left(\begin{array}{l}
i \\
2
\end{array}\right)+|U|-i\right]>|\tilde{E}|-1-\left[\left(\begin{array}{c}
\tilde{K} \\
2
\end{array}\right)+|U|-\tilde{K}\right]=\alpha^{I}
$$

for $\tilde{K} \geq 3$. Again a contradiction. Let us finally consider the case where $i=\tilde{K}$. Then by the above reasoning we find $\alpha_{q} \geq \alpha^{I}$ which together with $\alpha_{q} \leq \alpha^{I}$ implies that $\alpha_{q}=\alpha^{I}$ and hence the remaining knapsack capacity

$$
B^{I}-\frac{\tilde{K}}{\tilde{K}}-a(Q)-B^{I}+\left[1+\frac{\left(\begin{array}{c}
\tilde{K} \\
2
\end{array}\right)}{\tilde{K}^{3}}+a(Q)\right]=\frac{\left(\begin{array}{c}
\tilde{K} \\
2
\end{array}\right)}{\tilde{K}^{3}}
$$

must account for an increase in the solution of the maximization problem in (14) of at least $\left(\begin{array}{c}\tilde{K} \\ 2\end{array}\right)$. Hence, at least $\left(\begin{array}{c}\tilde{K} \\ 2\end{array}\right)$ vertices from $\tilde{E}$ must be included in the knapsack, and moreover, these vertices must have their predecessors in the graph in the knapsack. This can only be achieved if these vertices are in $E$, and hence we have found a clique of size $K$ in the graph $G$.

Although lifting variables for a PCKP is strongly NP-hard in general, in the special case where the precedence graph is a tree and the size of the coefficients in the given valid inequality is polynomially bounded, then lifting coefficients can be obtained in polynomial time.

Theorem 3.6 Given a PCKP for which the precedence graph is a tree, and a valid inequality with coefficients whose size is bounded by a polynomial in the size of the tree, then all lifting coefficients can be determined in polynomial time.

Proof. Lifting a variable requires solving a tree knapsack problem on a subtree of the original tree. Tree knapsack problems with possibly negative objective coefficients can be solved in pseudo-polynomial time $\mathcal{O}\left(n Q^{2}\right)$ by an extension of a standard dynamic programming algorithm for tree knapsacks (see for instance Jonson and Niemi ([12]), where $Q$ is an upper bound on the maximum value that can be achieved in the optimization problem. If all coefficients of the are polynomially bounded in the size of the original tree, then $Q$ is polynomially bounded, and therefore the tree knapsack problem can be solved in polynomial time.

\subsection{An Example}

Consider the example in Figure 1. Table 1 gives an example of both a minimal induced cover 


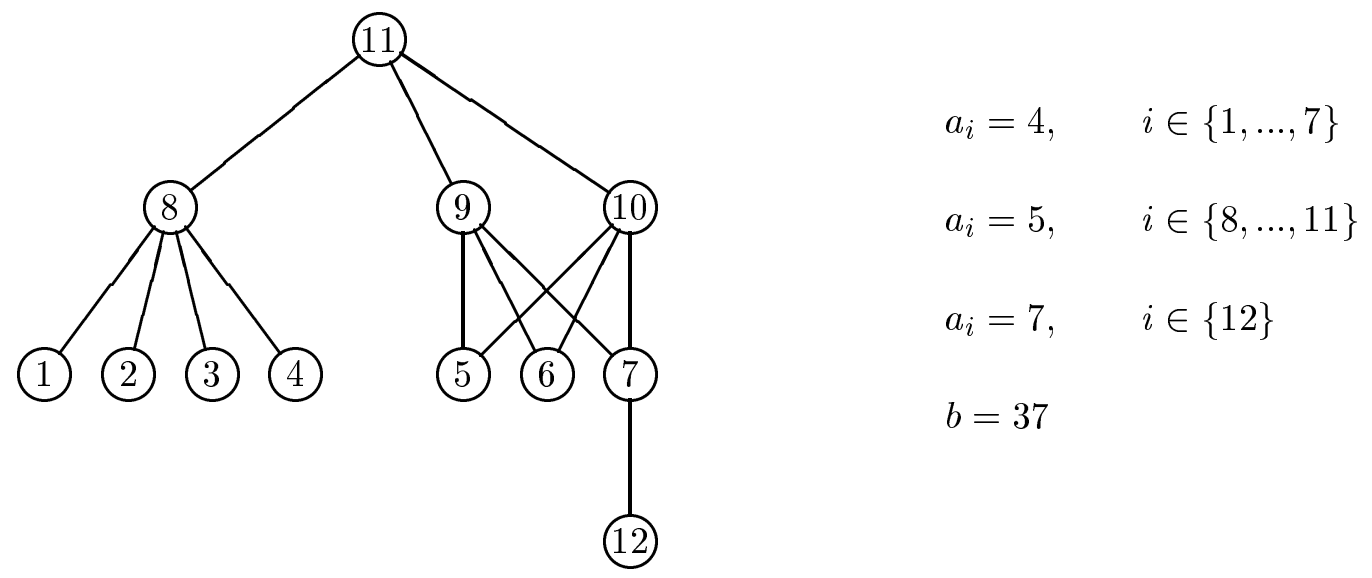

Figure 1: example.

for which two different PFRS-orders are stated and a $(1, \mathrm{k})$-configuration for which only two valid inequalities out of the total set as defined by (16) are listed. The resulting facet-defining inequalities are listed below. The combinatorial interpretation of the predecessors of a minimal induced cover can be seen from this table, which also illustrates that different PFRS-orders can lead to different facets. The reader may note that many more covers and $(1, \mathrm{k})$-configurations are present in the problem instance (see also Section 5).

\begin{tabular}{|l||r|r|l||r|}
\hline$C$ & $t$ & type & $Z(r)$ & lifting order \\
\hline \hline$\{1,2,5,6,7\}$ & - & MIC & - & $8,9,10,11,3,4,12$ \\
$\{1,2,5,6,7\}$ & - & MIC & - & $8,10,9,11,3,4,12$ \\
$\{1,2,5\}$ & 12 & $(1,2)-$ conf & $\{1,2\}$ & $7,8,9,10,11,3,4,6$ \\
$\{1,2,5\}$ & 12 & $(1,2)-$ conf & $\{1,2,5\}$ & $7,8,9,10,11,3,4,6$ \\
\hline
\end{tabular}

Table 1: A minimal induced cover and $(1, \mathrm{k})$-configuration for problem instance in Figure 1.

Resulting facet-defining inequalities for Table 1:

$$
\begin{aligned}
& x_{1}+x_{2}+x_{5}+x_{6}+x_{7}+\left(1-x_{8}\right)+2\left(1-x_{9}\right) \quad+\left(1-x_{11}\right)+x_{12} \leq 4 \\
& x_{1}+x_{2}+x_{5}+x_{6}+x_{7}+\left(1-x_{8}\right) \quad+2\left(1-x_{10}\right)+\left(1-x_{11}\right)+x_{12} \leq 4 \\
& \begin{array}{lllllll}
x_{1}+x_{2} & +\left(1-x_{8}\right) & +\left(1-x_{11}\right) & +x_{12} & \leq & 2
\end{array} \\
& x_{1}+x_{2}+x_{5}+\left(1-x_{8}\right)+\left(1-x_{9}\right) \quad+\left(1-x_{11}\right)+2 x_{12} \leq \quad 3
\end{aligned}
$$




\section{K-covers}

In this section, we discuss valid inequalities arising from K-covers. Although this class of inequalities is a direct generalization of minimal induced covers, it is not always immediately clear for which subset of the polytope the corresponding valid-inequalities are facet-defining. One way to obtain facets for the PCKP-polytope would be to follow two steps: first, the exact polytope for which the valid inequality is facet-defining could be determined and next, the same lifting procedure as mentioned in Section 3 could be applied. In this section, we show that if the first step is skipped, and a different lifting procedure is applied, again facets for the PCKP-polytope are obtained.

Definition 4.1 $C \subseteq V$ is a $K$-cover if

- $C$ is incomparable

- $\forall S \subseteq C$, with $|S|=K$ it holds that $a(T(S))>b$, but $a(T(S) \backslash\{i\}) \leq b, \forall i \in S$.

Let $C \subseteq V$ be a K-cover, then

$$
\sum_{i \in C} x_{i} \leq K-1
$$

is a valid inequality for the PCKP-polytope.

Figure 2 shows that if $C \subseteq V$ is a K-cover the subset $X_{P(C) \mid R(C)}$ can be empty, in which case the aforementioned lifting procedure cannot directly be applied to the variables in $P(C) \cup R(C)$. In this example $C=\{1,2,3,4\}$ is a 3 -cover, but $X_{P(C) \mid R(C)}=\emptyset$. In fact, the corresponding valid inequality is facet-defining for $\operatorname{conv}\left(X_{\{9,10,11\} \mid R(C)}\right)$.

Below we define a different lifting procedure which is still defined on $P(C) \cup R(C)$ and generates facet-defining inequalities. Note that the only difference between the two lifting procedures consists of the polytope over which the maximization problem is defined.

Definition 4.2 Let $C$ be a K-cover and $\pi$ be a PFRS-order for $C$ which represents the order of lifting variables. Let the lifting coefficient for a variable $j \in P(C)$ be determined by

$$
\alpha_{j}=K-1-\max _{\substack{x \in X: \\ x_{j}=0}}\left[\sum_{i \in C} x_{i}+\sum_{i \in P(C) \cap p^{\pi}(j)} \alpha_{i}\left(1-x_{i}\right)\right]
$$




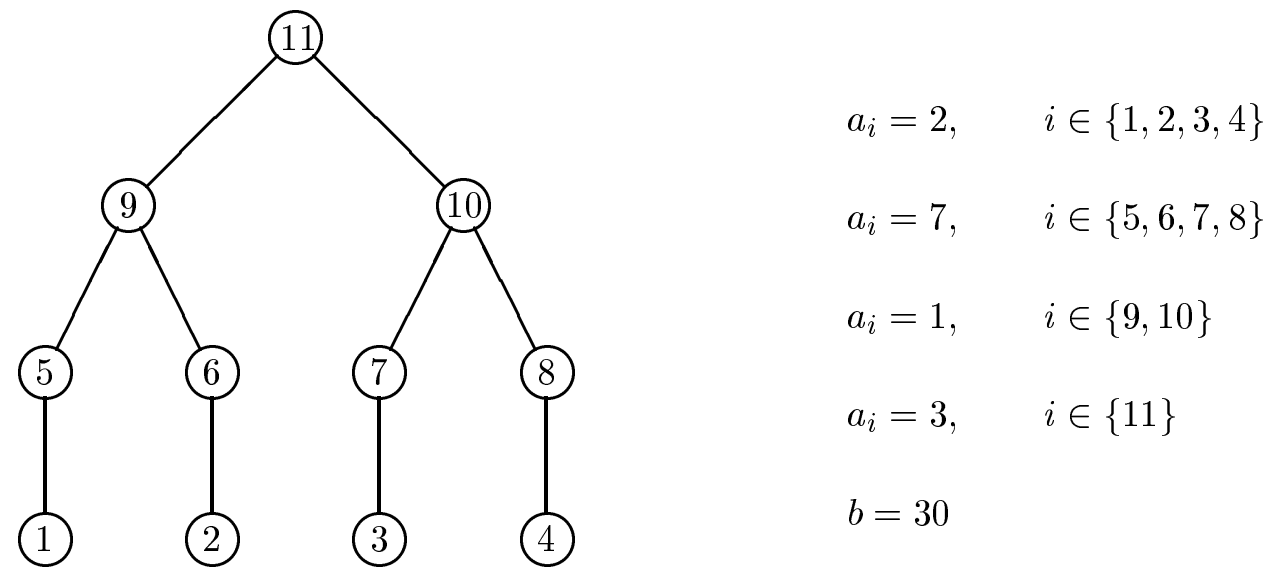

Figure 2: example k-cover

and for a variable $j \in R(C)$ by

$$
\alpha_{j}=K-1-\max _{\substack{x \in X: \\ x_{j}=1}}\left[\sum_{i \in C} x_{i}+\sum_{i \in P(C)} \alpha_{i}\left(1-x_{i}\right)+\sum_{i \in R(C) \cap p^{\pi}(j)} \alpha_{i} x_{i}\right]
$$

Theorem 4.1 Let

- $C \subseteq V$ be a K-cover

- $\pi$ be a PFRS-order for $C$

- lifting coefficients for variables in $P(C)$ determined according to (21)

- lifting coefficients for variables in $R(C)$ determined according to (22)

then the resulting inequality

$$
\sum_{i \in C} x_{i}+\sum_{i \in P(C)} \alpha_{i}\left(1-x_{i}\right)+\sum_{i \in R(C)} \alpha_{i} x_{i} \leq K-1
$$

a) is valid, and $\alpha_{i} \geq 0$, for all $i \in P(C) \cup R(C)$ 
b) defines a facet of the PCKP-polytope conv $(X)$.

Proof. Both validity and nonnegativity of the lifting coefficients can be proved easily using inductive arguments. We will prove the remainderof our claim by constructing $|V|-1$ linearly independent vectors (directions) in the face described by the inequality. These vectors are constructed as the difference of two vectors, both with the following properties:

(i) the vector satisfies the knapsack constraint;

(ii) the vector satisfies the precedence constraints;

(iii) the vector satisfies the lifted inequality at equality.

Properties (i) and (ii) imply that the vector is in $\operatorname{conv}(X)$, whereas property (iii) guarantees that the vector is in the face described by the inequality. For ease of notation, let $P^{0}(C)=\{j \in$ $\left.P(C) \mid \alpha_{j}=0\right\}$ and $P^{>}(C)=\left\{j \in P(C) \mid \alpha_{j}>0\right\}$. Note that for each $i \in P^{>}(C)$ the number of items in $C$ which are not successors of $i$ is less than or equal to $K-2$. If there were more than $K-2$ items in $C$ which are not successors of $i$, say set $S \subseteq C$ consisting of $K-1$ items, then the maximization problem in (21) for variable $x_{i}$ would have value at least $K-1$ since the items in $T(S)$ could be set to one. In other words, if $S \subset C$ contains $K-1$ elements, then $i$ is a predecessor of at least one element from $S$. This property is used at several occasions in the remainder of the proof.

Let $C=\{1, \ldots,|C|\}$. For $j=1, \ldots,|C|-1$ let $C^{j} \subseteq C \backslash\{j, j+1\}$ with $\left|C^{j}\right|=K-2$. Next, define

$$
\begin{aligned}
\phi^{j} & =x^{T\left(C^{j} \cup\{j, j+1\}\right) \backslash\{j+1\}} \\
\psi^{j} & =x^{T\left(C^{j} \cup\{j, j+1\}\right) \backslash\{j\}} \\
y^{j} & =\phi^{j}-\psi^{j}
\end{aligned}
$$

Then the vectors $y^{j}, j=1, \ldots,|C|-1$ are clearly linearly independent. Moreover, $\phi^{j}$ satisfies properties (i) and (ii) by definition: we take $K$ items from $C$ and all their predecessors; after that we remove one of the items from the $K$ items chosen. Clearly, the vector $\phi^{j}$ also satisfies property (iii): we have $K-1$ elements from $C$, and thus the first term of the left-hand side of (23) equals $K-1$. Since the other terms are nonnegative and the equation is valid, we must have that equality holds. For vector $\psi^{j}$, (i), (ii), and (iii) can be shown similarly.

For $j \in P^{0}(C)$, let $\theta^{j}$ be the vector for which the maximum in (21) is attained. Let $C^{j}=\{i \in$ $\left.C \mid \theta_{i}^{j}=1\right\}$. Thus, $\forall_{i \in T\left(C^{j}\right)} \theta_{i}^{j}=1$. Moreover, we may assume that $\forall_{i \notin T\left(C^{j}\right)} \theta_{i}^{j}=0$ : Clearly, this maintains feasibility with regard to both the knapsack constraint, and the precendence constraints. It remains to show that the maximum in (21) is not decreased. If $i \in C \backslash C^{j} \theta_{i}^{j}=0$ by definition of $C^{j}$. If $i \in P(C) \backslash T\left(C^{j}\right)$, setting $\theta_{i}^{j}=0$ can not have a decreasing effect on the maximum in (21), since $\left(1-x_{i}\right)$ becomes positive, and the objective coefficient of $\left(1-x_{i}\right)$ in (21) is nonnegative. If $i \in R(C)$, the objective coefficient of $x_{i}$ is zero in (21). 
We define

$$
\begin{aligned}
\phi_{i}^{j} & = \begin{cases}1 & i \in T\left(C^{j}\right) \cup T(j) \cup T\left(P^{>}(C) \cap s^{\pi}(j)\right) \\
0 & \text { otherwise }\end{cases} \\
\psi_{i}^{j} & = \begin{cases}1 & i \in T\left(C^{j}\right) \cup P(j) \cup T\left(P^{>}(C) \cap s^{\pi}(j)\right) \\
0 & \text { otherwise }\end{cases}
\end{aligned}
$$

Note that $\phi^{j}-\psi^{j}$ is the $j$-th unit vector, since $j \in T(j)$ but $j \notin T\left(C^{j}\right) \cup P(j) \cup T\left(P^{>}(C) \cap s^{\pi}(j)\right)$ (Note that $\theta_{j}^{j}=0$, see (21) ). It remains to show that $\phi$ and $\psi$ satisfy (i), (ii), and (iii). To show that the knapsack constraint is satisfied, we construct an extension of $\phi$ that does so. Let $\bar{C}^{j}$ be an extension of $C^{j}$ with $K-1$ elements from $C$. If $j \in T\left(\bar{C}^{j}\right)$ take an arbitrary $i \in C \backslash \bar{C}^{j}$. Otherwise choose $i \in C \backslash \bar{C}^{j}$ such that $j$ is a predecessor of $i$. By definition of $K$-covers, the set $T\left(\bar{C}^{j} \cup\{i\}\right) \backslash\{i\}$ satisfies the knapsack constraint. Clearly, $T\left(C^{j}\right)$ and $T(j)$ are subsets of $T\left(\bar{C}^{j} \cup\{i\}\right) \backslash\{i\}$. Furthermore, $P^{>}(C) \subset T\left(\bar{C}^{j}\right)$, since $T\left(\bar{C}^{j}\right)$ contains $K-1$ elements from $C$. Thus, $P^{>}(C) \cap s^{\pi}(j) \subset T\left(\bar{C}^{j}\right)$, and thus $T\left(P^{>}(C) \cap s^{\pi}(j)\right) \subset T\left(\bar{C}^{j}\right)$, and $T\left(\bar{C}^{j}\right) \subseteq T\left(\bar{C}^{j} \cup\{i\}\right) \backslash\{i\}$.

The precedence constraints hold by construction of $\phi^{j}$.

To show that (iii) is satisfied by $\phi^{j}$, we show that $\phi^{j}$ obtains the same value in the maximum of $(21)$ as $\theta^{j}$, i.e., $K-1$. This is true because $\phi^{j}$ is an extension of $\theta^{j}$ with elements from $s^{\pi}(j) \cup\{j\}$. This trivially holds for $P^{>}(j) \cap s^{\pi}(j)$ and $\{j\}$. It also holds for the predecessors of both sets, i.e., $T\left(P^{>}(j) \cap s^{\pi}(j)\right)$ and $T(j)$ by the definition of $\pi$. Since elements from $s^{\pi}(j)$ have no contribution to the maximum of $(21) \phi^{j}$ and $\theta^{j}$ have the same value. Next, the terms in the maximum of (21) are a subset of the terms in (23) with a value of $K-1$. The remaining terms are nonnegative, and hence, by validity of the inequality, therefore zero. Thus, (23) is satisfied at equality. Similar arguments show that (i), (ii), and (iii) hold for $\psi$.

Next, let $j \in P^{>}(C)$, and let $\theta^{j}$ be the vector for which the maximum in (21) is attained. W.l.o.g. assume that $\theta_{i}^{j}=0$ for $i \in R(C)$ and for $i \in P(C)$ such that $i$ is not a predecessor of an element in $C$ which is set to one. As indicated in the above, we can again extend the solution $\theta_{i}^{j}$ to a solution $\psi^{j}$, in which all variables in $T\left(P^{>}(C) \cap s^{\pi}(j)\right)$ are included in the knapsack. Next, let $\phi^{j}$ be any vector with $K-1$ elements in $C$ equal to one, and $\phi_{i}^{j}=0$, for $i \in R(C)$. Analogously as in the above, one can verify that $\psi^{j}$ and $\phi^{j}$ satisfy properties (i)-(iii). Define $y^{j}=\phi^{j}-\psi^{j}$, then $y_{j}^{j}=1$ and $y_{i}^{j}=0$, for $i \in P^{>}(C) \cap s^{\pi}(j)$ and $i \in R(C)$.

Finally, for $j \in R(C)$, let $\theta^{j}$ be the vector for which the maximum in (22) is attained. W.l.o.g., assume that $\theta_{i}^{j}=0$, for $i \in R(C) \cap s^{\pi}(j)$. Let $C^{j} \subset C$ with $\left|C^{j}\right|=K-1$. Next, define

$$
\begin{aligned}
\phi^{j} & =\theta^{j} \\
\psi^{j} & =x^{T\left(C^{j}\right)} \\
y^{j} & =\phi^{j}-\psi^{j}
\end{aligned}
$$


Then both $\phi^{j}$ and $\psi^{j}$ satisfy properties (i)-(iii). Finally, $y_{j}^{j}=1$ and $y_{i}^{j}=0$ for $i \in s^{\pi}(j)$.

We leave it to the reader to verify that the $|V|-1$ vectors as defined in the above are linearly independent.

The following example shows that Theorem 4.1 does not necessarily hold if we allow for more general lifting orders.

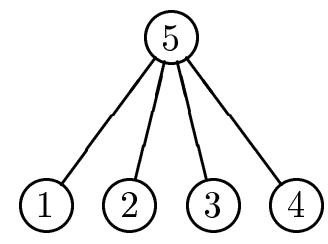

$$
\begin{array}{ll}
a_{i}=4, & i \in\{1, \ldots, 4\} \\
a_{i}=2, & i \in\{5\} \\
a_{i}=10, & i \in\{6\} \\
b=10 &
\end{array}
$$

Figure 3: example 2.

Now the set $\{1,2,3,4\}$ is a 3 -cover and hence

$$
\sum_{i=1}^{4} x_{i} \leq 2
$$

is a valid inequality. If we first lift variable $x_{6}$ according to (22) and then $x_{5}$ accoding to (21) we obtain the inequality

$$
\sum_{i=1}^{4} x_{i}+2 x_{6} \leq 2
$$

which is valid but not facet-defining for $\operatorname{conv}(X)$.

\section{Computational Results and Concluding Remarks}

To gain insight in the effectiveness of the proposed facets in this paper, reconsider the example of Subsection 3.4. In this small example 36 covers were found, which by applying different PFRSorders led to a total of 63 diferent facet-defining inequalities. Furthermore seven $K$-covers were found (11 different facets), and 24 (1,k)-configurations (leading to 100 different facets).

In the five problem instances in Table 2 nodes in the same "layer" of the graph are given the same, but randomly chosen, objective function coefficient. For each of these 5 problem instances, 
the value of the LP-relaxation, the IP-value and the value of the LP-relaxation after adding all 174 facets to the description were computed.

\begin{tabular}{|c|r|r|r|r|}
\hline problem & LP-value & LP + facets & IP-value & \%gap closed \\
\hline \hline obj1 & 49.3 & 47.8 & 46 & $45 \%$ \\
obj1a & 57.0 & 51.8 & 48 & $58 \%$ \\
obj1b & 104.0 & 104.0 & 100 & $0 \%$ \\
obj1c & 242.0 & 235.9 & 227 & $41 \%$ \\
obj1d & 270.6 & 244.6 & 225 & $57 \%$ \\
\hline
\end{tabular}

Table 2: Computational results for problem instance in Figure 1.

These results indicate that the effect of the valid inequalities may be significant. Problem instance obj1b represents a situation where nodes in layer three (i.e. nodes 1 through 7 ) have a low objective coefficient compared to the other nodes.

Next, 9 objective functions in which each node is given a random objective coefficient were generated. Different ranges of objective coefficients were tested. Table 3 reports on the computational results for these problem instances.

\begin{tabular}{|c|r|r|r|r|}
\hline problem & LP-value & LP + facets & IP-value & \%gap closed \\
\hline \hline obj2 & 124.0 & 119.1 & 119 & $97 \%$ \\
obj3 & 116.0 & 110.3 & 110 & $95 \%$ \\
obj4 & 104.6 & 97.0 & 97 & $100 \%$ \\
obj5 & 179.8 & 176.0 & 176 & $100 \%$ \\
obj6 & 231.7 & 228.3 & 226 & $60 \%$ \\
obj7 & 233.7 & 223.7 & 222 & $85 \%$ \\
obj8 & 274.0 & 251.4 & 248 & $87 \%$ \\
obj9 & 53.5 & 51.5 & 51 & $80 \%$ \\
obj10 & 43.2 & 41.0 & 41 & $100 \%$ \\
\hline
\end{tabular}

Table 3: Computational results for problem instance in Figure 1.

The results show that a large proportion of the gap can be closed by including the facets proposed in this paper. In fact, only PFRS-orders were considered in the tests, hence more valid inequalities can be included by allowing for more general lifting ordersas indicated in this paper. Finally we state some remarks on possible future research directions.

Firstly, note that problem instance obj1c shows that for certain problem instances the facetdefining inequalities discussed in this paper are not very useful. Direct generalizations of other well-known classes of valid inequalities for regular knapsack problems could of course form a fruitful area for future research. Next, to incorporate such inequalities into a branch-andcut procedure, the separation problem must be addressed. Again, generilazations of separation heuristics for ordinary cover inequalities can be investigated. Thirdly, the difference in definition of a minimal induced cover between on the one hand the definition as being used in this paper 
and by Boyd ([4]), and on the other hand the definition employed by Park and Park ([15]) deserves more research. Finally, the lifting procedure as proposed in Section 4 is only proven to be a valid procedure for valid inequalities arising from K-covers. In fact, in the proof detailed information from the definition of a K-cover is used. The question arises whether these lifting ideas can also be used for more different and/or more general classes of valid inequalities.

\section{References}

[1] E.H. Aghezzaf, T.L. Magnanti, and L.A. Wolsey. Optimizing constrained subtrees of trees. Mathematical Programming, 71:113-126, 1995.

[2] E. Balas. Facets of the knapsack polytope. Mathematical Programming, 8:146-164, 1975.

[3] E. Balas and E. Zemel. Facets of the knapsack polytope from minimal covers. SIAM Journal of Applied Mathematics, 34:119-148, 1978.

[4] E.A. Boyd. Polyhedral results for the precedence constrained knapsack problem. Discrete Applied Mathematics, 41:185-201, 1993.

[5] G. Cho and D.X. Shaw. A depth-first dynamic programming algorithm for the tree knapsack problem. Technical report, School of Industrial Engineering, Purdue University, 1994.

[6] G. Cho and D.X. Shaw. Limited column generation for local access telecommunication network design - formulations, algorithms, and implementation. Working paper, School of Industrial Engineering, Purdue University, 1995.

[7] Y. Crama. Combinatorial optimization models for production scheduling in automated manufacturing systems. In 14th European Conference on Operations Research, pages 237259, 1995.

[8] H. Crowder, E. Johnson, and M. Padberg. Solving large-scale zero-one linear programming problems. Operations Research, 31(5):803-834, 1983.

[9] M. R. Garey and D.S. Johnson. Computers and intractability. Freeman and Company, N.Y., 1979.

[10] D. Hartvigsen and E. Zemel. The complexity of lifted inequalities for the knapsack problem. Discrete Applied Mathematics, 39:113-123, 1992.

[11] S.H. Hwan and W. Shogan. Modeling and solving an FMS part selection problem. International Journal of Production Research, 27:1349-1366, 1989.

[12] D.S. Johnson and K.A. Niemi. On knapsacks, partitions and a new dynamic programming technique for trees. Mathematics of Operations Research, 8:1-14, 1983.

[13] J.W. Mamer and W. Shogan. A constrained capital budgeting problem with applications to repair kit selection. Management Science, 33:800-806, 1987. 
[14] G.L. Nemhauser and L.A. Wolsey. Integer and Combinatorial Optimization. Wiley, N.Y., 1988.

[15] K. Park and S. Park. Lifting cover inequalities for the precedence constrained knapsack problem. Discrete Applied Mathematics, 72:219-241, 1997.

[16] D.X. Shaw. Reformulation and column generation for several telecommunication network design problems. In Proceeding of the 2nd International Telecommunication Conference, Nashville, Tennessee, 1994.

[17] D.X. Shaw and G. Cho. A branch-and-bound procedure for the tree knapsack problem. Research memorandum 94-11, School of Industrial Engineering, Purdue University, West Lafayette, Indiana, 1994.

[18] K.E. Stecke and I. Kim. A study of part type selection approaches for short-term production planning. International Journal of Flexible Manufacturing Systems, 1:7-29, 1988.

[19] E. Zemel. Easily computable facets of the knapsack polytope. Mathematics of Operations Research, 14(4):760-764, 1989. 